\title{
Enseñanza de las ciencias desde el punto de vista del constructivismo orgánico
}

\section{Science education from the organic constructivism's point of view}

\author{
Nicolás Marín Martínez \\ Universidad de Almería \\ nmarin@ual.es
}

RESUMEN • Una visión del significado y su relación con el significante, desde una perspectiva constructivista, permite desarrollar una novedosa propuesta para la enseñanza de las ciencias. Este desarrollo es posible con el apoyo en un modelo de aprendizaje coherente con el constructivismo orgánico.

PALABRAS CLAVE: enseñanza de las ciencias; constructivismo orgánico; significado y significante; tipos de aprendizaje.

SUMMARY • An overview of signified and its relationship with the significant, from a constructivist perspective, allows to develop a new proposal for teaching science. This development is possible with the support in a learning model consistent with organic constructivism.

KEYWORDS: science education; organic constructivism; signified and significant; types of learning. 


\section{INTRODUCCIÓN}

Es curioso constatar en la literatura de Didáctica de las Ciencias Experimentales (DCE), revisando las diferentes propuestas más significativas para mejorar la enseñanza de las ciencias, que no se haya enfatizado con mayor contundencia la distinción entre significado y significante. Esta distinción es importante, por ejemplo, para entender y analizar cómo se transmite el conocimiento del docente al alumno de ciencias, un asunto nada trivial desde una visión constructivista.

También, esta distinción permite comprender o discutir mejor cuestiones tales como que: a) lo escrito en el libro de texto no es más que un cúmulo de significantes sin apenas significado para el alumno, $b$ ) lo que llega de la explicación del docente al alumno son meros significantes carentes de significado, c) para muchos conceptos de ciencias reconstruir su significado solo es posible con actividades socialmente compartidas y empíricamente recontextualizadas, $d$ ) extender o ampliar el significado es una cuestión de grados incluso para el experto de ciencias, o $e$ ) el alumno no puede operar adecuadamente con significantes de ciencias si previamente no les asigna un significado.

Lo anterior, junto a otras diferenciaciones propias del conocimiento individual, por ejemplo, entre conocimiento implícito y explícito o entre lo lógico y lo psicológico, es lo que se pretende argumentar y justificar en este trabajo desde el marco teórico del constructivismo orgánico. Tras un cambio de perspectiva para la enseñanza de las ciencias, se desarrolla un modelo de enseñanza, denominado el alumno como aprendiz, que considera un conjunto de orientaciones didácticas fundamentadas en detalles epistemológicos y psicológicos sobre los conocimientos de ciencias y del alumno, que si bien muestran analogías, también presentan importantes diferencias (Marín, 2011, 2003a).

\section{EL CONSTRUCTIVISMO ORGÁNICO. CREACIÓN DE ESTRUCTURAS QUE ASIGNAN SIGNIFICADO}

El constructivismo es la posición más consensuada para analizar asuntos de conocimiento, y es la que se usará como marco para establecer las diferencias entre significante y significado, llevar esta distinción a los conocimientos de ciencias y del alumno y analizar cómo se generan las estructuras del alumno que asignan significado.

Las propuestas didácticas que se muestran más adelante se fundamentan en una versión constructivista denominada constructivismo orgánico $(\mathrm{CO})$, que originalmente hinca sus raíces en la teoría de la equilibración piagetiana (por ejemplo, Piaget, 1977, 1978) y por tanto se desmarca de otras opciones constructivistas con compromisos empiristas o mecanicistas. El CO experimenta un proceso de ampliación con revisiones actualizadas (Delval, 1997, 2002; Pascual-Leone, 1979, 1983; Pozo, 1989, 2003) y, más recientemente, integra nuevas formulaciones sobre organización cognitiva y aprendizaje (Aparicio-Serrano y Pozo, 2006; Pecharromán y Pozo, 2008; Pecharromán, Pozo, Mateos y Pérez Echeverría, 2009; Pozo, 1996, 2003; Pozo y Flores, 2007; Pozo, Scheuer, Mateos y Echeverría, 2006; Pozo, Scheuer, Pérez Echeverría, et al., 2006; Rodrigo y Correa, 1999).

Una exposición más extensa del CO se puede encontrar en anteriores publicaciones (Marín, 2003b, 2005, 2010a). Aquí se muestra una versión centrada en dos cuestiones determinantes para la argumentación posterior: a) diferenciación entre significado y significante y $b$ ) tipos de aprendizaje relevantes en la enseñanza de las ciencias. 


\section{A) Distinción entre significado y significante. El problema de la asignación de significados}

Las nociones de significante y significado dependerán del marco epistemológico y teórico elegido. Un marco realista puede ver innecesaria la distinción, de forma que con la llegada de un significante al sujeto llegaría también su significado. El CO percibe que desde el exterior solo pueden llegar significantes carentes de significado y que este solo se puede asignar en el interior del sujeto, por lo que la distinción es fundamental.

Una palabra, un dibujo, la percepción de un objeto, una imagen, un símbolo y, en general, todo aquello que llega a los sentidos del sujeto desde el exterior es significante. El significado es lo que hace sentir o entender al sujeto el significante, lo cual depende de sus vivencias, sentimientos, lecturas, charlas con los demás, conocimientos anteriores y, en general, de la totalidad biológica del sujeto (Castilla del Pino, 2000; Marina, 1998). El significado es asignado a un significante en el interior del sujeto, por lo que se afirma que es "un atributo exclusivamente humano" (Marina, 1998).

Piénsese en el proceso para asignar significado cuando se implantó el euro. En un inicio, los números carecían de significado y fue poco a poco, con actividades tales como comprar, convertir a la antigua moneda o comparar con el esfuerzo en ganarlo, cuando los números fueron tomando peso, dimensión, sentido, etc., en definitiva, las cantidades se van llenando de significado para cada sujeto en función de sus interacciones semióticas y sensomotrices con el medio.

Por el reiterado uso del vínculo significado-significante en las relaciones interpersonales, pareciera que finalmente se hace automático, y de ahí, es usual pensar intuitivamente que van adosados uno al otro o que tienen una identidad indisoluble. Desde la posición del CO, el significante toma un significado en el interior del sujeto (Delval, 1997; Glasersfeld, 1993). El significado tiene un componente individual, fruto de las peculiaridades de la historia personal de cada cual, pero sobre todo tiene un componente social, fruto de la intensa interacción con los demás y de la semiótica cultural. La etimología (evolución del significado de las palabras) muestra que en el lenguaje tanto el significado como el vínculo con su significante son arbitrarios y regulados socialmente en una dialéctica construcción en la mente de cada individuo (Marín, 2010a).

Quizá la principal razón por la que el constructivismo insiste en separar interior y exterior del sujeto es porque percibe conocimiento y realidad como entidades o categorías diferentes que no admiten correspondencia y, por tanto, imposibles de comparar (Pozo, 1989). También por esto se distingue entre realidad externa y construida. Con la primera el sujeto interactúa, pero no puede acceder a ella para comparar directamente con su conocimiento. La segunda, ubicada en el interior del sujeto que la construye, es a la que se puede acceder. La realidad construida cambia con el progreso cognitivo (Marín, 2003b). La sensación realista de que el conocimiento representa y se corresponde con la realidad es menos adecuada que considerarlo fruto del proceso de adaptación (Claxton, 1987; Delval, 1997). Lo que capta el sujeto al interactuar con el medio es una interpretación de los órganos sensitivos y cognitivos, no el medio en sí.

La distinción constructivista entre significante y significado corre paralela a la que se hace también entre información y conocimiento. La información es la versión externa del conocimiento, en forma de significantes usualmente registrados en un soporte físico (signos, símbolos, significantes verbales, percepciones, sensaciones, acciones, etc.). Esta información, para que sea conocimiento, requiere un sujeto que conozca el código y sus reglas gramaticales, disponer de los conocimientos previos necesarios para poder asignarle significado y, finalmente, acomodarla para que se convierta en conocimiento. Así pues, lo que circula entre las personas es información, y solo puede convertirse en conocimiento en la mente del sujeto (Delval, 2002; Pozo, 2003). 


\section{B) Tipos de aprendizajes significativos para la enseñanza de las ciencias}

La visión orgánica del aprendizaje que maneja el CO postula que la construcción cognitiva nueva se crea como una reacción o regulación orgánica ante desequilibrios, perturbaciones o conflictos del sistema cognitivo cuando interactúa con su exterior e interior. Esta visión del aprendizaje se aleja de otras en las que se admite cierto grado de correspondencia entre las condiciones externas de enseñanza y lo que se aprende (Delval, 1997, 2002; Marín, 2003b).

La unidad básica de la organización cognitiva es el esquema de conocimiento, con la doble función de ser acumulador orgánico y regulador adaptativo de las interacciones entre sujeto y medio (Piaget, 1978). En un primer nivel y construidos como reacción a los desequilibrios de las interacciones externas, estarían los esquemas específicos sensibles a los contenidos de la interacción natural o social (Marín, 1994). En un nivel de mayor abstracción, construidos desde procesos de reflexión con los materiales cognitivos del primer nivel, estarían ubicados otros esquemas cognitivos de mayor grado de generalidad, entre los que hay que destacar, por su importancia para la enseñanza de las ciencias, los esquemas operatorios (Piaget, 1977a).

Buscando un orden con cierta intención sistemática para la exposición de los distintos tipos de aprendizaje, se diferencian inicialmente dos grupos, según si las interacciones del sujeto se dan con su medio externo o interno. Esta distinción se fundamenta en que los primeros son aprendizajes más fáciles de lograr y los procesos constructivos se realizan en el seno de esquemas específicos (Marín, 1994, 1998); mientras que los segundos suponen procesos constructivos que requieren una implicación cognitiva y afectiva del sujeto a largo plazo (Karmiloff-Smith y Elsabbagh, 2006; Marchand, 2001; Pascual-Leone, 1983; Pozo, 2007).

Las interacciones externas aportan información a través de una diversidad de significantes. Se distinguen, según la dificultad para crear aprendizaje, dos tipos: interacciones sensomotrices, en las que los significantes que aporta la experiencia directa del contacto físico con los objetos del medio proporcionan "datos brutos"; e interacciones semióticas, en las que el sujeto interactúa con signos y símbolos culturales cuyos significados están socialmente compartidos (Delval, 1997; Marín, 2005, 2010a; Rodrigo, Rodríguez y Marrero, 1993):

- En el primer caso, el proceso constructivo por el que la información externa se convierte en conocimiento que enriquece un esquema específico se denominará integrar. Primero debe tomar significado adecuado la información externa (asimilación) y después el esquema sufre un proceso de reajuste para integrar el nuevo contenido (acomodación). El proceso de integrar, lejos de ser simple, lineal y acumulativo, es un proceso orgánico más o menos tortuoso que requiere tanteos, ajustes, rectificaciones, ensayo, etc. (Piaget, 1978), lo que conlleva cierto tiempo de implicación afectiva y cognitiva por parte del sujeto.

- En el segundo caso, cuando se conoce el significado de los significantes socialmente compartidos, usualmente palabras, es posible enriquecer el esquema de un modo más sencillo. El significado social tiene mayor capacidad adaptativa al recoger un gran cúmulo de experiencias pasadas; no obstante, el sujeto lo adquiere progresivamente en su continua interacción social. Aunque en este caso también se dan los procesos de asimilación y acomodación, la integración no es tan tortuosa como en el caso de significantes brutos fruto de la experiencia directa, ni tan fácil que suponga apropiación directa. La organización conceptual del entramado semiótico cultural, socialmente compartida, facilita el enriquecimiento de significados individuales (Marín, 2010a).

Para entender los procesos constructivos por la interacción del sujeto con el espacio semiótico interpersonal, donde se da la interacción entre lo individual y lo social, el CO introduce en esta zona de interacción un nuevo constructo formado por un "sistema simbiótico que aglutina la red semiótica de 
una sociedad y la comunidad que la profesa y puede asignarle significados" (Marín, 2010a). Este sistema se va constituyendo de tal modo que tanto los significantes como los vínculos con los significados se construyen en un espacio socialmente compartido donde la necesidad de comunicación genera un contexto de tensión dialéctica que favorece las regulaciones sociales, así, a) se incrementan los recursos del sistema semiótico compartido, $b$ ) se disminuye el margen de tolerancia del significado (diferencia entre su núcleo compartido y la zona más individual (Marina, 1998), c) se crean nuevos vínculos entre el sistema semiótico y los significados, etc. En pocas palabras, el sistema semiótico-comunidad aglutina lo social y lo individual creando en la mente del sujeto nuevos progresos cognitivos.

En la evolución cognitiva, primero se desarrollan los esquemas por las interacciones sensomotrices. Luego, estos dan soporte cognitivo al juego simbólico del sujeto por el que se inicia un proceso de diferenciación entre significantes y significados que desemboca en la adquisición del lenguaje. A partir de este momento el sujeto enriquece los esquemas desde el acervo cultural (Delval, 2002; Piaget \& Inhelder, 1984).

Las interacciones internas suponen procesos de reflexión sobre contenidos y procedimientos cognitivos. Las construcciones cognitivas de este procesamiento interior en diferido se suelen dar en periodos largos de tiempo. El interés, la motivación o los deseos del sujeto pueden explicar en buena medida la vuelta reiterada para retomar el problema, el conflicto o el esfuerzo cognitivo. Estas interacciones pueden terminar en nuevas construcciones tras la intensa implicación cognitiva y afectiva del sujeto. Por procesos internos se perciben al menos tres tipos de construcciones:

- Explicitar. Procesos constructivos ligados a la explicitación de esquemas. Estos inicialmente poseen naturaleza procedimental e implícita. Poco a poco, el sujeto toma cierto grado de conciencia de su existencia, llegando a hacerlo representativo usando los signos y símbolos socialmente compartidos (Karmiloff-Smith y Elsabbagh, 2006; Marín, 2010; Piaget, 1976; Pozo et al., 2010).

- Operar. Cuando el objeto de reflexión son las mecánicas de regulación de los esquemas que buscan el nuevo equilibrio ante una perturbación o conflicto cognitivo, en un proceso reiterado de largo plazo, se pueden originar esquemas operatorios (Marín, 2005; Piaget, 1978), proceso que se denomina abstracción reflexiva (Piaget, 1977b).

- Formalizar. Conlleva procesos de reflexión sobre los contenidos cognitivos cuando estos se encuentran en formato explícito a través de una semiótica socialmente compartida. Esto hace más fácil la reflexión interior, pero también la exterior, por ejemplo, en foros o lecturas. En este contexto, el sujeto genera estructuras cognitivas nuevas que permiten dar significado a los conceptos más abstractos de la cultura, tales como: empirismo, realismo, constructivismo, etc. (en el contexto de la epistemología), o prima de riesgo, inflación, recesión (en el contexto de las finanzas) (Pozo, 2003). Estos esquemas formales serían explícitos al sujeto.

La metacognición se entiende como la reflexión consciente sobre objetos y procesos cognitivos (Organista Díaz, 2005) fruto de la constante autorregulación que actúa en todos los niveles de la actividad cognitiva (Flores Ochoa, 2000; Piaget, 1976). Los tres procesos anteriores suponen, de un modo u otro, metacognición: explicitar-tomar conciencia del esquema-, operar-reflexionar sobre las mecánicas de regulación-y formalizar -reflexionar sobre los objetos cognitivo.

De la fuerte participación del sistema afectivo del alumno en las diferentes mecánicas constructivas, habría que resaltar dos de estas que son determinantes para entender mejor las orientaciones didácticas posteriores:

- Integrar (asimilar y acomodar) no es lo mismo que comprender (asimilar sin acomodar). Esto último no supone la retención a largo plazo de lo primero. Ir de comprender a integrar requiere 
la implicación afectiva del sujeto para volver de forma reiterada sobre la información externa que pone resistencia, a pesar de los sucesivos conflictos cognitivos que genera.

- Operar requiere la implicación afectiva del alumno para volver recurrentemente a reflexionar sobre las mecánicas que compensan las perturbaciones cognitivas.

Del repertorio expuesto de construcciones cognitivas se puede deducir y desarrollar una amplia gama de orientaciones didácticas para la enseñanza de las ciencias, que se exponen a continuación.

\section{ORIENTACIONES DIDÁCTICAS PARA LA ENSEÑANZA DE CIENCIAS. MODE- LO DE ENSEÑANZA DEL ALUMNO COMO APRENDIZ}

A los modelos de enseñanza de las ciencias que se deducen de una visión adecuada de cómo se construye el conocimiento de ciencias se los ha denominado modelos basados en la analogía del alumno como cientifico-AcC- (Marín, 2011), y contienen propuestas que han sido muy populares, tales como la del cambio conceptual, la de las concepciones alternativas o la de enseñanza por investigación. Ahora que las orientaciones didácticas se deducen de una visión adecuada sobre cómo se construye el conocimiento del alumno (procesos constructivos, integrar, operar, explicitar o formalizar), el modelo que las integra se denominará el alumno como aprendiz (AcA). Las orientaciones didácticas importantes que se deducen de los distintos procesos constructivos del aprendiz, que constituyen el modelo AcA, son las siguientes:

\section{A) Diferenciar y enfatizar el significado frente al significante. El primero, ligado a los esque- mas cognitivos, requiere mayor tiempo de aprendizaje}

La distinción entre significado y significante, junto a la mecánica de la asignación de significados, explicarían en general que los alumnos apenas retengan unos pocos contenidos de ciencias de los muchos que se les enseñan y que lo poco retenido apenas puedan aplicarlo en cuestiones prácticas que requieran una solución. Una enseñanza centrada en significantes apenas da oportunidad al alumno para que asigne significados adecuados, dado que, en bastantes ocasiones, carece de un sustrato cognitivo de esquemas para que lo haga. Se requiere algo más si se pretende que el alumno integre los contenidos académicos.

Para contenidos de ciencias tales como reciclaje, contaminación o conservación del agua, en los que el alumno puede asignar significados adecuados, aunque muy básicos, es posible tener resultados aceptables con recursos tales como explicaciones, debates y búsqueda de información en internet. Pero no siempre el alumno tiene los esquemas de conocimiento suficientemente desarrollados como para dar significado a unos significantes que suele desconocer.

En el contexto de la clase de ciencias, apenas se da tiempo al alumno para llenar de significado a los significantes, que en muchos casos solo se retienen por memorización, y los vínculos con situaciones prácticas y concretas son escasos, con lo que se crea un conocimiento plano, imposibilitado para la transferencia cognitiva (Pozo y Gómez Crespo, 1998). Por el contrario, los significados que usualmente asigna el alumno a conceptos propios del escenario cotidiano, al construirse desde una diversidad de interacciones (físicas, semióticas y vicarias) en periodos de tiempo amplios, poseen una carga procedimental importante para ser aplicados en contextos prácticos.

El conocimiento de ciencias, para quien no conoce el código en el que está registrado, o es lego en la materia, la información significa bien poco o nada. Los entramados de información de ciencias, es decir, la versión declarativa de ciencias, se convierten en conocimiento gracias a que son profesados, 
consensuados, gestionados y mantenidos por un colectivo de individuos -comunidad científica- que lo usa para buscar soluciones más eficaces al conjunto amplio de problemas asociados a las teorías (Marín, 2010b).

Entonces, ¿cómo percibe el alumno el libro de texto de ciencias? Responder a esto solo es posible siguiendo la traza de los significantes grabados en documentos, pues los significados se perdieron en las mentes de los ilustres científicos. Habría que remontarse unos siglos atrás, cuando el contexto cognitivo, socioeconómico y problemático del momento, diferente al actual, genera inquietudes entre los científicos, y fruto de sus indagaciones llegan a un nuevo conocimiento, que dejan escrito a generaciones posteriores. En esta versión declarativa desaparece el contexto problemático, los procesos de la indagación y el conocimiento original de los autores. Después, otros expertos de la comunidad se dedican a decantar, filtrar, modificar, formalizar, sintetizar, etc., el conocimiento científico heredado, con lo que van dejando sucesivas versiones declarativas más alejadas aún de la original, dado que se van ajustando a nuevos contextos. Finalmente, un experto hace una nueva versión declarativa para consumo escolar, que sería el libro de texto de ciencias.

El alumno, en su lectura del libro de texto de ciencias, se encuentra con un compendio de significantes sin la contextualización original, sin los procesos de investigación que los originaron y sin los procesos de formalización, síntesis y transposición que dieron lugar a la versión que intenta leer. Además, lejos de la mente experta que pudiera darle significado, carece de semejante estructura cognitiva. Ver así el libro de texto, por la evolución que experimenta en el tiempo de los significantes, permite entender mejor que el alumno lo perciba en ocasiones como un sinsentido (pensar por momentos en el concepto de inercia), muestre rareza, le cueste comprenderlo o le sea casi imposible su vinculación con sus conocimientos cotidianos.

Otra clave importante para entender la adquisición del significado de un concepto de ciencias es distinguir formalmente entre su comprensión y su extensión. Todo concepto, y en particular los de ciencias, como producto del pensamiento lógico, se caracteriza por su comprensión (conjunto de caracteres o atributos que lo definen) y su extensión (conjunto de objetos o conceptos de orden inferior a los que se refiere). Por ejemplo, dado un conjunto de objetos geométricos, si la comprensión es "objetos con tres lados", la extensión estará formada por todos y cada uno de los objetos triangulares que hay en el conjunto.

Las mecánicas de aprendizaje de la comprensión y la extensión son diferentes. La primera se identifica con la definición del concepto a través de otros significantes (Marina, 1998). Si el alumno no tiene esquemas para dar significado a los significantes, algo frecuente en los conceptos de ciencias, se verá abocado a memorizarlos, pero si los tiene, es posible que una hábil explicación del profesor logre, en breve espacio de tiempo, la comprensión del concepto. Aun así, la comprensión no supone la integración efectiva en algún esquema de conocimiento (Marín, 2005). Ahora bien, aunque el camino es más largo, si se da al aprendiz un tiempo amplio para interactuar con los objetos empíricos de la extensión del concepto, podrá ir enriqueciendo el significado por procesos de integración, y va a adquirir suficientes procedimientos como para posibilitar su aplicabilidad (Pozo y Pérez Echeverría, 2009).

La interacción del alumno con la extensión de un concepto supone en la práctica de clase el desarrollo de actividades procedimentales dirigidas a ampliar sus significados. Aprender de las actividades procedimentales, elaborar ideas o conceptos de la experiencia o asignar significados adecuados a los datos empíricos, suponen procesos cognitivos no lineales que no pueden ser asimilados a una secuencia lógica. En consecuencia, las actividades procedimentales no deben marcar un ritmo preestablecido y lineal, sino más bien un tipo de enseñanza abierta, en la que pueda entrar en juego la duda, la rectificación, el ensayo y error o los tanteos tanto empíricos como mentales del sujeto. El diseño de las actividades debe contemplar un margen de actuación del alumno tal que evite restringir en exceso 
sus respuestas - por ejemplo, que solo tenga que afirmar o negar- y, a la vez, evitar lo contrario: una formulación tan abierta que el alumno no tenga información suficiente para desarrollar la actividad.

Así pues, no basta con memorizar o comprender los contenidos de ciencias; es necesario también integrarlos a los esquemas y dotarlos de una buena carga procedimental para garantizar su transferencia a contextos prácticos (Pozo y Gómez Crespo, 1998). Para ello es preciso que el alumno interactúe con la parte extensional de los conceptos (referentes concretos), creando vínculos sólidos con la comprensión del concepto.

\section{B) Recontextualizar. Diseñar las actividades con variaciones contextuales y, en cada contex- to, realizar variaciones relevantes e irrelevantes de los diferentes factores que intervienen en la actividad}

La orientación didáctica A (hacer interactuar al alumno con la extensión de los conceptos de ciencias) se retoma ahora con mayor decisión para profundizar en ella. El CO percibe insuficiente que se pueda aprender de una sola experiencia aislada; solo sería así desde una visión empirista que asuma que el dato empírico es conocimiento (Marín, 2002). La extensión de un concepto se refiere a una diversidad de objetos o situaciones, que si no se pueden abordar en la enseñanza con intención sistemática sí que habrá que hacerlo con una muestra significativa para que haya aprendizaje.

La recontextualización se refiere al intento de dar significados a los significantes del contenido haciendo que el alumno interactúe con los contextos más prácticos y empíricos de este, de un modo estructurado, con la intención de obtener información o buscar respuestas a un determinado contexto problemático inicial. Esto permitirá ir llenando de significado el contenido, pero también adquirir suficientes recursos procedimentales, de modo que el nuevo conocimiento, una vez adquirido, se pueda aplicar a una diversidad de situaciones prácticas (Pozo y Pérez Echeverría, 2009). Esta idea es especialmente relevante si se intenta fomentar competencias. Las estrategias para cubrir el componente práctico y el empírico de un contenido de ciencias pueden ser las siguientes:

- Estrategias de variación contextual ( $V c$ ). Consisten en crear cierta sistemática en las situaciones y objetos que se van a considerar sobre el contenido que enseñar. Los contextos deben ser potencialmente interesantes para el alumno y deben estar formulados a modo de cuestiones o problemas a fin de marcar un sentido a las actividades: su intención será buscar soluciones principalmente mediante el esfuerzo cognitivo del alumno, con la ayuda puntual del profesor. Como mínimo habría que considerar dos o tres contextos prácticos por contenido, buscando que estos sean diferentes entre sí.

- Estrategias de variación del grado de complejidad o dificultad (Vd). Para cada contexto, hay que diseñar un gradiente de complejidad o dificultad que vaya desde situaciones sencillas, en las que la interacción entre ellos se rige por relaciones causales elementales, por ejemplo de contigüidad espacial o temporal (Pozo y Gómez Crespo, 1998), hasta otras más complejas, en las que la geometría de los objetos, el número de estos y sus interacciones se hacen más complicadas. Este gradiente dará más garantías de desarrollo de los esquemas por integración de los nuevos datos.

- Estrategias de variaciones relevantes e irrelevantes $\left(V_{r}, V_{i}\right)$. Suponen una visión dinámica de la interacción del sujeto con los contextos prácticos de cada actividad. La intención es que el alumno perciba regularidades, excepciones, relaciones causa-efecto, irrelevancias, etc. Se denomina variación relevante a las modificaciones de un factor de la situación o contexto que produce una alteración relevante o modifica significativamente un resultado. Variación irrelevante será por tanto aquella que al experimentarla un determinado factor no supone ningún tipo de alteración relevante en la situación o en el resultado. Podría parecer que las variaciones irrelevantes carecen 
de utilidad didáctica; sin embargo, permiten al alumno tomar conciencia de la existencia de factores irrelevantes, incluso cuando anteriormente los percibiera significativos.

La recontextualización ofrece la oportunidad de desarrollar los esquemas específicos, puesto que al ejercitarse repetidamente permiten generalizarse a la diversidad de contextos y posibilitan pequeños reajustes del esquema para ir acomodando la diversidad de datos. El resultado que se pretende es que el alumno adquiera los contenidos de un modo más flexible, más rico en significados y en recursos procedimentales de aplicación práctica y, por tanto, con mayores posibilidades de ser transferidos a otros contextos. El coste, claro está, es la necesidad de tiempos de aprendizaje mayores para cada contenido.

\section{C) Crear un contexto de enseńanza expectante y comprometido para fomentar en el alum- no nuevas construcciones a través de desequilibrios y reequilibrios}

Si las orientaciones de enseñanza anteriores detallan cómo diseñar las actividades con cierta sistemática inter- e intracontextual, ahora se dan nuevas orientaciones para diseñar actividades a fin de aumentar las posibilidades de desequilibrios y reequilibrios cognitivos. Se hace necesario implicar al alumno cognitiva y afectivamente como único modo de afrontar los desequilibrios con cierta garantía de éxito. Se trata de crear un espacio de tensión dialéctica entre él y las actividades y entre él y los demás, en grupo, con toda la clase y con el profesor. Se trata de crear un contexto de enseñanza expectante, atractivo y comprometido que permita no solo los conflictos cognitivos, resolviendo problemas o compartiendo significados con los demás, sino también el tener ganas e interés para afrontarlos en la búsqueda de nuevos reequilibrios cognitivos (Marín, 2005).

Los contenidos académicos deben experimentar las suficientes transformaciones como para que resulten atractivos a los alumnos, solo así sería posible lograr dicha implicación. La actitud del profesor también debe cambiar para que sea más motivadora, lejos del rigor académico o de imposiciones en aras al supuesto valor intrínseco de los contenidos.

Obsérvese que no se dará desequilibrio en el alumno si: $a$ ) el sujeto no quiere entrar en el juego de la asimilación y acomodación de lo nuevo por indolencia, desmotivación, falta de interés, etc., $b$ ) producido este, el sujeto vuelve a su anterior equilibrio rechazando o negando la perturbación (predominio de su esquema por encima de otras evidencias), c) lo que se propone como elemento de perturbación es para él algo irrelevante y d) el dato es tan novedoso que es imposible su asimilación a un esquema.

Si se consigue la perturbación, conflicto cognitivo o desequilibrio, la posibilidad de que la experiencia se convierta en integración dependerá de que el nuevo dato resulte más o menos familiar para alguno de sus esquemas o de que esté interesado o motivado para adquirirlo.

Para ayudar al alumno en la reequilibración, se requiere diseñar actividades encaminadas a invitarlo a reflexionar en determinadas direcciones que pueden conducir a la solución, ya que la reequilibración, al ser un proceso interno al sujeto, requiere un tiempo de reflexión para reajustar sus esquemas iniciales con el fin de incorporar los nuevos conocimientos (acomodación). En ningún caso se darían soluciones o información significativa al comienzo, pues inhibiría sus esfuerzos cognitivos y por tanto las posibles reconstrucciones cognoscitivas necesarias para llegar a la reequilibración. Este es el fundamento principal de la enseñanza por descubrimiento dirigido, basada en la teoría de la equilibración de Piaget (1978).

El control del docente para crear perturbaciones y reequilibrios es bastante menor que si solo se pretende que el alumno memorice o comprenda, pudiendo establecer condiciones de enseñanza "precisas" que posibiliten un desequilibrio. Por otra parte, es importante cuidar las condiciones afectivas para que el alumno entre con interés y ganas al desarrollo de las actividades.

Algunas sugerencias para diseñar actividades que aumenten la posibilidad de lograr conflictos cognitivos son: 
- Crear una situación inicial y solicitar a los alumnos que expliquen o expresen manipulativamente cómo sería posible lograr unos objetivos u otros.

- Plantear un problema y buscar una solución a través de una pequeña investigación. Habría una versión manipulativa de este diseño en la que el problema se plantea en un contexto material y para encontrar la respuesta se deben manipular objetos.

- Hacer previsiones a partir de una situación inicial y, después de la experiencia, solicitar explicaciones al alumno al confrontar sus previsiones iniciales con los resultados de la experiencia.

Piénsese en las características de una adivinanza: atractiva, sugerente y todos quieren dar con la solución. Así se debe diseñar la actividad que pretenda implicar cognitiva y afectivamente al alumno. Se logra mayor implicación cognitiva en el alumno si las actividades se desarrollan en contextos sociales donde se dan interacciones intragrupales e intergrupales y en todo momento se solicita que se vayan explicitando los progresos.

\section{D) Resignificar. Fomentar los procesos de reflexión en un ambiente de compromiso, dedica- ción e intensa interacción semiótica en el aula}

Las nuevas construcciones por reflexión, como en ocasiones anteriores, requieren la implicación cognitiva y afectiva del alumno a fin de insistir sobre el problema planteado, el conflicto y el esfuerzo cognitivo, incluso cuando los resultados esperados no son inmediatos. Si integrar una información nueva en un esquema requiere una dedicación importante en tiempo de enseñanza, mayor aún será para las construcciones por procesos de reflexión. Por suerte, se puede usar intencionada e intensivamente la interacción semiótica de clase para facilitar los procesos de reflexión, estrategia, complementaria a la de recontextuar (más centrada en las interacciones sensomotrices), que se llamará resignificar (más centrada en las interacciones semióticas).

Los periodos de enseñanza para que se den las nuevas construcciones por reflexión son largos. En estos se precisa que el docente, con un actuar en clase coherente con los procesos constructivos por reflexión, desarrolle un plan de actividades que se aplique una y otra vez para que haya diversidad de contenidos. Para ello, será necesario que dichas actividades resulten atractivas para el alumno, y mejor que sea por motivos endógenos (interés por el tema) que exógenos (necesidad de aprobar). Se considerarán las siguientes orientaciones didácticas:

Para favorecer la toma de conciencia autónoma se pueden diseñar actividades que soliciten la consecución de un objetivo y en las que se puedan percibir los resultados de las acciones o la toma de decisión del alumno. La actividad es mejor que sea manipulativa, y si es intelectiva la toma de decisiones debe concretarse, registrándola o explicándola a los demás, para que se pueda ver su resultado. Para potenciar la toma de conciencia, la actividad se hace en grupo, siendo objeto de reflexión en un primer momento el vínculo acción-resultado, para después reflexionar sobre los medios entre la acción y los resultados.

- El aula es un espacio que posibilita una intensa interacción social, por lo que resulta un lugar óptimo para explicitar los contenidos implícitos del alumno, y donde, interaccionando con materiales adecuados o con compañeros de su grupo de trabajo o en debates de clase, puede ir poniendo palabras adecuadas a dichos contenidos, lo cual puede conllevar en primer lugar una necesaria toma de conciencia. Fomentar el uso de la argumentación razonada (Driver, Osborne y Newton, 2000; Jiménez Aleixandre y Díaz de Bustamante, 2003; Sardá y Sanmartí, 2000) para defender los diferentes puntos de vista o aquellos inventados oportunamente por el profesor sería otro modo efectivo de lograr la necesaria implicación del alumno, no solo para afrontar 
con decisión los momentos de conflicto cognitivo, sino también para animar a la explicitación de las ideas del alumno. Tras las actividades propias de la toma de conciencia autónoma, una vez establecidos los consensos intragrupales, se puede abrir un debate intergrupal más amplio, en colaboración con el profesor, en el que se defiendan los diferentes puntos de vista con argumentos o evidencias que permitan ir explicitando con recursos semióticos compartidos los contenidos implícitos individuales a la vez que se enriquecen con el aporte de nuevos significados. Las interacciones entre iguales contienen un importante potencial para el aprendizaje (Carugati \& Mugny, 1988).

- Para fomentar las construcciones operatorias se perciben dos líneas de orientaciones: la primera, ya comentada, sugiere estrategias de variación relevante e irrelevante, en las que la reflexión constante sobre los incrementos y decrementos de las variables o factores que intervienen en la situación obliga al alumno a ir estructurando operatoriamente dichas variables; la segunda propone estrategias de confrontación y conflicto cognitivo para que, aplicadas a una diversidad de contenidos de ciencias, el alumno pueda reflexionar sobre las mecánicas de compensación y reequilibración y, de este modo, desde las regularidades que pueda entrever, una y otra vez, pueda construir la operatoria subyacente (Inhelder y Piaget, 1972). Esta reflexión se favorece en el entorno social de la clase, de modo que posiblemente permita acelerar las construcciones operacionales, las cuales han mostrado ser muy importantes para el aprendizaje de contenidos de ciencias (Lawson, 1993; Niaz, 1991; Shayer y Adey, 1993). Piénsese que detrás de muchos razonamientos del alumno, de sus habilidades hipotético-deductivas, de su capacidad de argumentar y, en general, de sus capacidades procedimentales, están sus operaciones mentales (Inhelder, Sinclair, \& Bovet, 1974; Lawson, 1994; Niaz, 1991; Shayer \& Adey, 1993).

- Para fomentar los procesos que se han denominado formalizar, hay que tener en cuenta que son reflexiones sobre contenidos cognitivos explícitos y conscientes. Para esto se precisan actividades intra- e intergrupales en las que los objetos de interacción sean materiales semióticos específicos del conocimiento matemático, filosófico, epistemológico, etc., y se combinen periodos largos de reflexión interior con interacciones externas semióticas en debates, lecturas o elaboraciones escritas. Ahora bien, si no hay un contexto cognitivo básico, creado a partir de referentes empíricos o prácticos que permita dar significado adecuado a los significantes de dichos entramados semióticos, el avance estará lleno de parcelas sin sentido.

En resumen, las orientaciones didácticas expuestas permiten desarrollar una enseñanza procedimental sobre los contenidos específicos de ciencias donde las adquisiciones por integración favorecen la transferencia cognitiva. A la vez, permiten desarrollar en el alumno capacidades procedimentales que actúan como herramientas cognitivas necesarias para reflexionar, controlar y ejecutar mejor su conocimiento (Karmiloff-Smith, 1994; Marina, 2012; Piaget, 1976; Pozo, 2003), para mejorar sus interacciones con el medio usando mecánicas hipotético-deductivas (Inhelder y Piaget, 1972) o para procesar la información de un modo más eficiente (Case, 1983; Pascual-Leone, 1983; Rumelhart y Ortony, 1982). Si enseñar contenidos específicos, en general, es difícil, la adquisición de los contenidos procedimentales es aún más y, consecuentemente, las estrategias de enseñanza son más exigentes, pues deben mantenerse coherentemente, en plazos de tiempos largos, aplicadas a una diversidad amplia de contenidos y en contextos específicos (Marín, 2005). 


\section{SÍNTESIS Y CONCLUSIONES}

El esquema 1 recoge la idea general de la propuesta AcA que se ha desarrollado:

Esquema 1.

Modelo del alumno como aprendiz

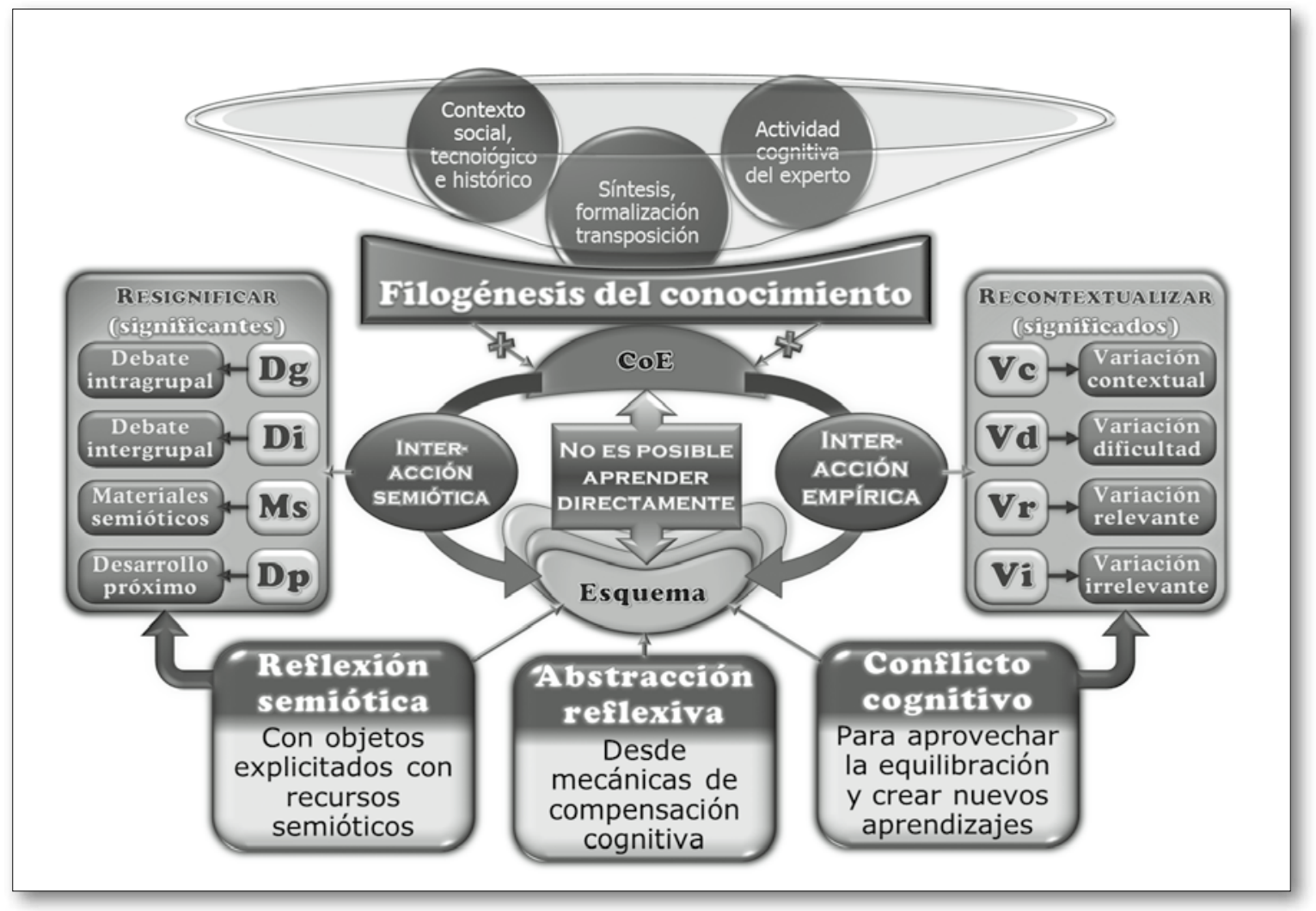

El esquema parte de la imposibilidad, en general, de transferir el contenido objeto de enseñanza ( $\mathrm{CoE})$ al alumno usando únicamente la vía de los significantes: es necesario crear un sustrato básico de esquemas que puedan ir dando significado al material semiótico de ciencias. Las orientaciones didácticas A, B, C y D muestran dos líneas maestras que pretenden integrar el contenido de ciencias que hay que enseñar en los esquemas del aprendiz:

- Recontextualizar el contenido de ciencias con contextos prácticos que permitan al alumno darle el sentido adecuado a la vez que enriquece sus esquemas con las nuevas experiencias. Las orientaciones didácticas basadas en las mecánicas de conflicto cognitivo y la reequilibración son las más indicadas para esta fase.

- Resignificar. En un primer momento se trataría de compartir significados al realizar la recontextualización en grupos de trabajo (intragrupo) y buscar consensos a través de debates entre grupos (intergrupo). Después se primaría la interacción semiótica con libros, profesor e internet. Las orientaciones didácticas basadas en las mecánicas constructivas por reflexión (explicitar, operar y formalizar) serían las más adecuadas para aplicar en esta fase. 
Consiste en ir creando nuevas construcciones cognitivas para que el sustrato de esquemas de conocimiento vaya enriqueciéndose hasta el punto de que el alumno adquiera la capacidad de asignar significados adecuados a la versión declarativa de ciencias.

El docente tiene la tarea de diseñar los procesos de enseñanza anteriores y participar como organizador y dinamizador de las actividades a la vez que va moderando los debates con su opinión y planteando cuestiones y dudas que inviten a los alumnos a reflexionar e ir explicitando sus ideas.

En general, las primeras actividades deben invitar al esfuerzo cognitivo del aprendiz ante las cuestiones problemáticas planteadas al inicio, y solo en las últimas será en las que se aporte información significativa para dar soluciones, es decir, la secuencia básica de la enseñanza por descubrimiento dirigido (Marín, 2005).

Los modelos AcC se fundamentan en el supuesto paralelismo entre el conocimiento de ciencia y del alumno, lo que da legitimidad a fundamentar sus orientaciones didácticas en algún aspecto o teorización de la construcción del conocimiento de ciencias. Sin embargo, junto a ciertos paralelismos entre ambos conocimientos también existen notables diferencias (Marín, 2003a). Por esta razón, las orientaciones didácticas del modelo AcA presentan novedades en relación con el modelo AcC principalmente donde los procesos cognitivos del conocimiento individual son distintos a los de ciencias, como puede ser la asignación de significados, el desarrollo de los esquemas cognitivos, los procesos de equilibración del conflicto cognitivo o los procesos de reflexión; por ejemplo:

- La orientación didáctica A se ocupa de extraer consecuencias didácticas atendiendo y centrando la atención en la construcción de los significados en el alumno. A sería bien distinta si se deduce de la asignación de significados en el contexto de ciencias, ya que al ser un conocimiento socialmente compartido requiere ser lo más explícito posible en un formato de red conceptual. De este modo se facilita al científico la asignación del significado a un concepto por su posición en la red y sus relaciones en dicho entramado con otros conceptos.

- La orientación didáctica B centra la atención en el constructo "esquema de conocimiento" como la entidad capaz de asignar significados y convertir en conocimiento los contenidos de ciencias. La enseñanza dirige sus esfuerzos a enriquecer los esquemas por procesos de integración y abre un espacio al uso de estrategias de variación contextual, del grado de complejidad y de factores relevantes e irrelevantes para diseñar actividades, así como una interacción alumnoactividad basada en procesos tentativos característicos del aprendizaje individual que muestran poca o ninguna semejanza con los procedimientos propios de la metodología científica.

- La orientación didáctica $\mathrm{C}$ ofrece una visión de la enseñanza de las ciencias diseñando estrategias de confrontación y conflicto cognitivo para que el alumno realice esfuerzos de reequilibración, algo que pudiera parecer semejante a lo que propone el cambio conceptual, pero en realidad es bien distinto, pues mientras este último propone una sustitución cognitiva imposible y estrategias para debilitar ideas previas, $\mathrm{C}$ busca el proceso de asimilación y acomodación, la extensión asimiladora del esquema o procesos de diferenciación para originar nuevos esquemas (Marín, 2011; Marín, 1999).

- La orientación didáctica D, al fundamentarse en procesos tales como la toma de conciencia, la abstracción reflexiva o la formalización semiótica, tan específicos del conocimiento individual, no tiene uso en las diferentes teorizaciones sobre la construcción del conocimiento de ciencias, y esto es así porque las construcciones cognitivas del experto individual no quedan registradas en los escritos históricos, tan solo se registran los hechos y descubrimientos significativos tamizados por la comunidad, y es que lo psicológico no existe en la epistemología de la ciencia (Chalmers, 1984).

Se pueden consultar más exhaustivamente otras ventajas del modelo AcA respecto a propuestas alternativas para la enseñanza de las ciencias ya publicadas en anteriores trabajos: 
- Revisión de los supuestos epistemológicos de los modelos del alumno como científico (AcC) sobre los conocimientos de ciencias y del alumno para descubrir que las deficiencias de estos sobre asuntos ligados al aprendizaje pueden ser superados por AcA (Marín, 2011).

- Cualidades de la visión orgánica del aprendizaje y de la enseñanza de ciencias frente a propuestas del cambio conceptual fundamentado en la psicología cognitiva (Marín, 2011).

- Valoración del potencial de CO, asumiendo procesos constructivos de aprendizaje social frente a otras propuestas usuales de la enseńanza de las ciencias (Marín, 2010a).

\section{BIBLIOGRAFÍA}

Aparicio-Serrano, J. A. y Pozo, J. I. (2006). De fotógrafos a directores de orquesta: las metáforas desde las que los profesores conciben el aprendizaje. En J. I. Pozo, N. Scheuer, M. P. Pérez Echeverría, M. Mateos, E. Martín y M. de la Cruz (eds.), pp. 265-288. Barcelona: Graó.

Arrieta, X., y Marín, N. (2002). Del experimento al concepto. Revista Encuentro Educacional, 9(2), pp. 125-146.

Carugati, F. y Mugny, G. (1988). La teoría del conflicto sociocognitivo. En Psicología social del desarrollo cognitivo, pp. 79-94. Barcelona: Anthropos.

Case, R. (1983). El desarrollo intelectual: una reinterpretación sistemática. En M. Carretero y J. A. García Madruga (eds.), pp. 339-362. Madrid: Alianza Editorial.

Castilla del Pino, C. (2000). Teoría de los sentimientos. Barcelona: Tusquets.

Chalmers, A. F. (1984). ¿Qué es esa cosa llamada ciencia? Madrid: Siglo XXI.

Claxton, G. (1987). Vivir y aprender. Madrid: Alianza.

Delval, J. (1997). Tesis sobre el constructivismo. En M. J. Rodrigo y J. Arnay (eds.), pp. 15-24. Barcelona: Paidós.

Delval, J. (2002). Vygotsky y Piaget sobre la formación del conocimiento. Investigación en la Escuela, 48, pp. 13-38.

Driver, R.; Osborne, J. F. y Newton, P. (2000). Establishing the norms of scientific argumentation in classrooms. Science Education, 48(3), pp. 287-312.

http://dx.doi.org/10.1002/(SICI)1098-237X(200005)84:3<287::AID-SCE1>3.3.CO;2-1

Flores OchoA, R. (2000). Autorregulación, metacognición y evaluación. Acción Pedagógica, 9(1), pp. 4-11.

Glasersfeld, E. von (1993). Introducción al constructivismo radical. En P. Watzlawick (ed.), pp. 20-27. Barcelona: Gedisa.

Inhelder, B. y Piaget, J. (1972). De la lógica del niño a la lógica del adolescente. Buenos Aires: Paidós.

Inhelder, B.; Sinclair, M. y Bovet, M. (1974). Aprendizaje y estructuras de conocimiento. Madrid: Morata.

Jiménez Aleixandre, M. P. y Díaz de Bustamante, J. (2003). Discurso de aula y argumentación en la clase de ciencias: Cuestiones teóricas y metodológicas. Enseñanza de las Ciencias, 21(3), pp. 359-370.

Karmiloff-Smith, A. (1994). Más allá de la modularidad. Madrid: Alianza.

Karmiloff-Smith, A. y Elsabbagh, B. (2006). Modularity of Mind and Language. In H. Brown (ed.), Encyclopedia of Language and Linguistics, vol. 8, pp. 218-224. Oxford: Elsevier.

Lawson, A. E. (1993). Deductive reasoning, brain maduration, and science concept acquisition: Are they linked? Journal of Research in Science Teaching, 30(9), pp. 1029-1051.

http://dx.doi.org/10.1002/tea.3660300904 
Lawson, A. E. (1994). Uso de los ciclos de aprendizaje para la enseñanza de destrezas de razonamiento científico y de sistemas conceptuales. Enseñanza de las Ciencias, 12(2), pp. 165-187.

Marchand, H. (2001). Some Reflections On PostFormal Thought The Genetic Epistemologist, 3(29). Disponible en: <http://www.piaget.org/GE/2001/GE-29-3.html\#item2>

Marín, N. (1994). Elementos cognoscitivos dependientes del contenido. Revista interuniversitaria de formación del profesorado, 20, pp. 195-208.

Marín, N. (1998). Contenidos procedimentales de Didáctica de las Ciencias Experimentales. Almería.

Marín, N. (1999). Delimitando el campo de aplicación del cambio conceptual. Enseñanza de las Ciencias, 17(1), pp. 79-92.

Marín, N. (2003a). Conocimientos que interaccionan en la enseñanza de las ciencias. Enseñanza de las Ciencias, 21(1), pp. 65-78.

Marín, N. (2003b). Visión constructivista dinámica para la enseñanza de las ciencias. Enseñanza de las Ciencias, extra, pp. 43-55.

Marín, N. (2005). La enseñanza de las ciencias en Educación Infantil. Granada: Grupo Editorial Universitario.

Marín, N. (2010a). Nuevas opciones constructivas en la teoría de Piaget sugeridas por las ideas de Vygotsky. EDUCyT, 1, pp. 57-74.

Marín, N. (2010b). Propuesta para compartir una base de datos de información bibliográfica (Bib) en Didáctica de las Ciencias. Revista Eureka sobre Enseñanza y Divulgación de las Ciencias, 7(3), pp. 613-635.

Marín, N. (2011). Evaluación de propuestas de cambio conceptual hechas desde la psicología cognitiva. Reflexiones sobre el aprendizaje de ciencias. Revista Eureka sobre Enseñanza y Divulgación de las Ciencias, 8(2), pp. 613-635.

Marín, N. y Cárdenas, F. A. (2011). Valoración de los modelos más usados en la enseñanza de las ciencias basados en la analogía "el alumno como científico". Enseñanza de las Ciencias, 29(1), pp. 35-46.

Marina, J. A. (1998). La selva del lenguaje. Barcelona: Anagrama.

Marina, J. A. (2012). La inteligencia ejecutiva. Barcelona: Ariel.

NiAZ, M. (1991). Correlates of formal operational reasoning: a neo-piagetian analysis. Journal of Research in Science Teaching, 28(1), pp. 19-40. http://dx.doi.org/10.1002/tea.3660280104

Organista Díaz, P. (2005). Conciencia y metacognición. Avances en Psicología Latinoamérica, 23, pp. 77-89.

Pascual-Leone, J. (I979). La teoría de los operadores constructivos. In J. Delval (ed.), pp. 208-228. Madrid: Alianza Universitaria.

Pascual-Leone, J. (1983). Problemas constructivos para teorías constructivas. En M. Carretero y J. A. García Madruga (eds.), pp. 363-392. Madrid: Alianza Editorial.

Pecharromán, I. y Pozo, J. I. (2008). Epistemologías intuitivas de los adultos: influencia de la edad, el nivel de instrucción y el dominio de conocimiento. Estudios de Psicología, 29(1)(3), pp. 245-272. http://dx.doi.org/10.1174/021093908786145412

Pecharromán, I.; Pozo, J. I.; Mateos, M. y Pérez Echeverría, M. P. (2009). Psicólogos ante el espejo: las epistemologías intuitivas de los estudiantes de psicología. Avances en Psicología Latinoamericana, 27(1), pp. 61-78.

Piaget, J. (1976). La toma de conciencia. Madrid: Morata.

Piaget, J. (1977a). Epistemología genética. Argentina: Solpin.

Piaget, J. (1977b). Lógica y psicología. Argentina: Solpin. 
Piaget, J. (1978). La equilibración de las estructuras cognitivas, "Problema central del desarrollo". Madrid: Siglo XXI.

Piaget, J. y Inhelder, B. (1984). Psicología del niño. Madrid: Morata.

Pozo, J. I. (1989). Teorías cognitivas del aprendizaje. Madrid: Morata.

Pozo, J. I. (1996). No es oro todo lo que reluce ni se construye (igual) todo lo que se aprende: contra el reduccionismo constructivista. Anuario de Psicología, 69, pp. 127-139.

Pozo, J. I. (2003). La adquisición de conocimiento. Madrid: Morata.

Pozo, J. I. (2007). Ni cambio ni conceptual: la reconstrucción del conocimiento científico como un cambio representacional. En J. I. Pozo y F. Flores (eds.). Madrid: Machado.

Pozo, J. I. y Flores, F. (2007). Cambio conceptual y representacional en el aprendizaje y la enseñanza de la ciencia. Madrid: Machado.

Pozo, J. I. y Gómez Crespo, M. A. (1998). Aprender y enseñar ciencia. Madrid: Ediciones Morata.

Pozo, J. I.; Martín, E.; Pérez Echeverría, M. P.; Scheuer, N.; Mateos, M. y De la Cruz, M. (2010). Ni contigo ni sin ti... Las relaciones entre cognición y acción en la práctica educativa. Infancia y aprendizaje, 33(2), pp. 179-184.

http://dx.doi.org/10.1174/021037010791114580

Pozo, J. I. y PÉrez Echeverría, M. P. (2009). Psicología del aprendizaje universitario: la formación en competencias. Madrid: Morata.

Pozo, J. I.; Scheuer, N.; Mateos, M. y Echeverría, M. P. P. (2006). Las teorías implícitas sobre el aprendizaje y la enseñanza. En J. I. Pozo, N. Scheuer, M. P. Pérez Echeverría, M. Mateos, E. Martín y M. De la Cruz (eds.), pp. 95-134. Barcelona: Graó.

Pozo, J. I.; Scheuer, N.; Pérez Echeverría, M. P.; Mateos, M.; Martín, E. y Cruz, M. D. la (2006). Nuevas formas de pensar la enseñanza y el aprendizaje. Barcelona: Graó.

Rodrigo, M. J. y Correa, N. (1999). Teorías implícitas, modelos mentales y cambio educativo. En J. I. Pozo y C. Monereo (eds.), pp. 75-86. Madrid: Aula XXI/Santillana.

Rodrigo, M. J.; Rodríguez, A. y Marrero, J. (1993). Las teorías implícitas: una aproximación al conocimiento cotidiano. Madrid: Visor.

Rumelhart, D. E. y Ortony, A. (1982). The representation of knowledge in memory. Infancia y aprendizaje, 20, pp. 115-158. http://dx.doi.org/10.1080/02103702.1982.10821949

SARdÁ, A. y Sanmartí, N. (2000). Enseñar a argumentar científicamente: un reto de las clases de ciencias. Enseñanza de las Ciencias, 18(3), pp. 405-422.

Shayer, M. y Adey, P. S. (1993). Accelerating the development of formal thinking in middle and high school students IV: three years after a two years intervention. Journal of Research in Science Teaching, 30(4), pp. 351-366.

http://dx.doi.org/10.1002/tea.3660300404 


\title{
Science education from the organic constructivism's point of view
}

\author{
Nicolás Marín Martínez \\ Universidad de Almería \\ nmarin@ual.es
}

Through an analysis based on the existing relationship between the signifier and the signified, it is possible to develop a new proposal for science-teaching. By establishing a conceptual differentiation between signifier and signified, it can be argued that: a) the content written in a text book is nothing more than a great number of signifiers, which have little meaning for the student, b) when a teacher provides an explanation, whatever gets through to the student are only signifiers with no meaning (they lack signifieds) for the students, which is why "reconstructing the signifieds" is only possible in science-related concepts through social activities which must be both shared and "recontextualized" empirically, c) amplifying the meaning of any science concept is a matter of grades or levels which never end, despite the level of expertise, d) it is not possible to work with science-related signifiers without previously having assigned a signified to such signifiers.

With fundaments taken mainly from history and philosophy of science, science-teaching models have been deducted from what is conceived as a correct representation of how scientific knowledge is constructed. Such methods have been designated as models founded on the analogy of the -student as scientist- (Marín, 2011). These models contain popular science teaching proposals such as conceptual change, misconceptions or learning through investigation. These science-teaching models are based on the parallelism between knowledge of science and student's knowledge. Such parallelism "allows" to found its didactic orientations in some aspect or theorization of the scientific knowledge construction.

In the context in which this work is developed a new science-teaching model is proposed which is deducted from a correct vision as to how the student's knowledge is constructed. This proposal is later on referred to as -student as apprentice-conformed by a series of didactic orientations found on epistemological and psychological aspects of the knowledge of science and the student's knowledge.

The didactic orientations of the-student as apprentice- model provide new approaches concerning the student as scientist- model, especially by arguing that the cognitive processes of the individual knowledge are differentiated from those of sciences (Marín, 2003a), for example, in the assignation of signifieds, the development of specific schemas, cognitive conflicts equilibration processes, or the cognitive reflection processes which make way to the creation and development of general schemas. 